\title{
EXPERIMENTAL DETERMINATION OF TEMPERATURES IN SPARK-GENERATED BUBBLES OSCILLATING IN WATER
}

\begin{abstract}
KAREL VOKURKA
Physics Department, Technical University of Liberec, Studentská 2, 46117 Liberec, Czech Republic

correspondence: karel.vokurka@tul.cz

ABstract. The surface temperatures of the plasma core in the final stages of the first contraction phase of spark-generated bubbles oscillating under ordinary laboratory conditions in a large expanse of water are determined experimentally. The measurement method is based on an analysis of the optical radiation from the bubbles and on the assumption that the plasma core is radiating as a black-body. It is found that the maximum surface temperatures of the plasma core range 4300-8700 K.
\end{abstract}

KEYWORDS: spark-generated bubbles; temperatures in bubbles; bubble oscillations.

\section{INTRODUCTION}

Bubble oscillations remain an important topic in fluid dynamics. While they are traditionally associated with erosion damage [1, 2], recent efforts have been aimed at medical applications, such as contrastenhancing in ultrasonic imaging [3] and shock wave lithotripsy [4]. Both spark-generated bubbles [2, [5] 7. 9,11] and laser-generated bubbles [1, 8, 12, 18] are very useful tools in experimental studies of free bubble oscillations.

The temperature in the interior of a bubble is a very important quantity, and it is necessary to know its variation with time in order to understand the physical processes, such as light emission, taking place in an oscillating bubble. However, it is very difficult to determine the temperatures in spark-generated and laser-generated bubbles experimentally. Until now, only a limited number of estimates based on spectral analysis of the light emitted from the bubbles have been reported, in [5, 8, 11, 12, 14, 15]. The difficulties associated with temperature measurements have their origin in the fact that the temperature in a bubble interior varies very rapidly in the final stages of the contraction phase, and the emitted light used for spectral analysis therefore has the form of a very short flash of light. Another difficulty in the measurements originates from the fact that experimental spark-generated bubbles have very low reproducibility (in terms of bubble size and intensity of oscillation). Although the reproducibility of lasergenerated bubbles may be better, it remains relatively low.

This paper will show that useful estimates of the instantaneous surface temperature of the plasma core in the bubble interior can be determined using the experimental method described in 19 . In this way, autonomous behaviour of the plasma can also be proved. The analysis presented here is devoted to free bubble oscillation under ordinary laboratory conditions in a large expanse of liquid. The results discussed here have been presented in a shortened form at a conference in Svratka [20].

\section{EXPERIMENTAL SETUP}

Freely oscillating bubbles were generated by discharging a capacitor bank via a sparker submerged in a laboratory water tank with dimensions of $6 \times 4 \times 5.5 \mathrm{~m}$ (length $\times$ width $\times$ depth). The experiments were performed in tap water at constant hydrostatic pressure $p_{\infty}=125 \mathrm{kPa}$, at room temperature $\Theta_{\infty}=292 \mathrm{~K}$, and far from any boundaries. The capacitance of the capacitor bank could be varied in steps by connecting 1-10 capacitors in parallel. Each of these capacitors had a capacitance of $16 \mu \mathrm{F}$. The capacitors were charged from a high-voltage source of $4 \mathrm{kV}$. An air-gap switch was used to trigger the discharge through the sparker. A schematic diagram of the experimental setup is given in Figure 1

Both the spark discharge and the subsequent bubble oscillations were accompanied by intensive optical radiation and acoustic radiation. The optical radiation was monitored by a detector, which consisted of a fiber optic cable, a photodiode, an amplifier, and an A/D converter. The input surface of the fiber cable was positioned in water at the same level as the sparker, at a distance of $0.2 \mathrm{~m}$ aside, pointing perpendicular to the sparker gap and the electrodes. A Hammamatsu photodiode type S2386-18L was positioned at the output surface of the fiber optic cable. The usable spectral range of the photodiode is 320 $1100 \mathrm{~nm}$. An analysis of the optical spectra given in the literature showed that the maximum temperatures in spark-generated and laser-generated bubbles range 5800-8150 K [5, 11, 15, 18]. Then, using the Wien and Planck Law, it can be verified that the spectral maxima of the optical radiation are within the photodiode bandpass, and that the prevailing part of the radiation is received by the detector. The load resistance of the photodiode was $75 \Omega$, so the rise time of the measured pulses is about 50 ns. A broadband amplifier $(0-10 \mathrm{MHz})$ was connected to the photodiode output terminals. The output voltage from the amplifier was recorded using a data acquisition board (National Instruments PCI 6115, 12 bit A/D converter) with a sampling frequency of $10 \mathrm{MHz}$. 


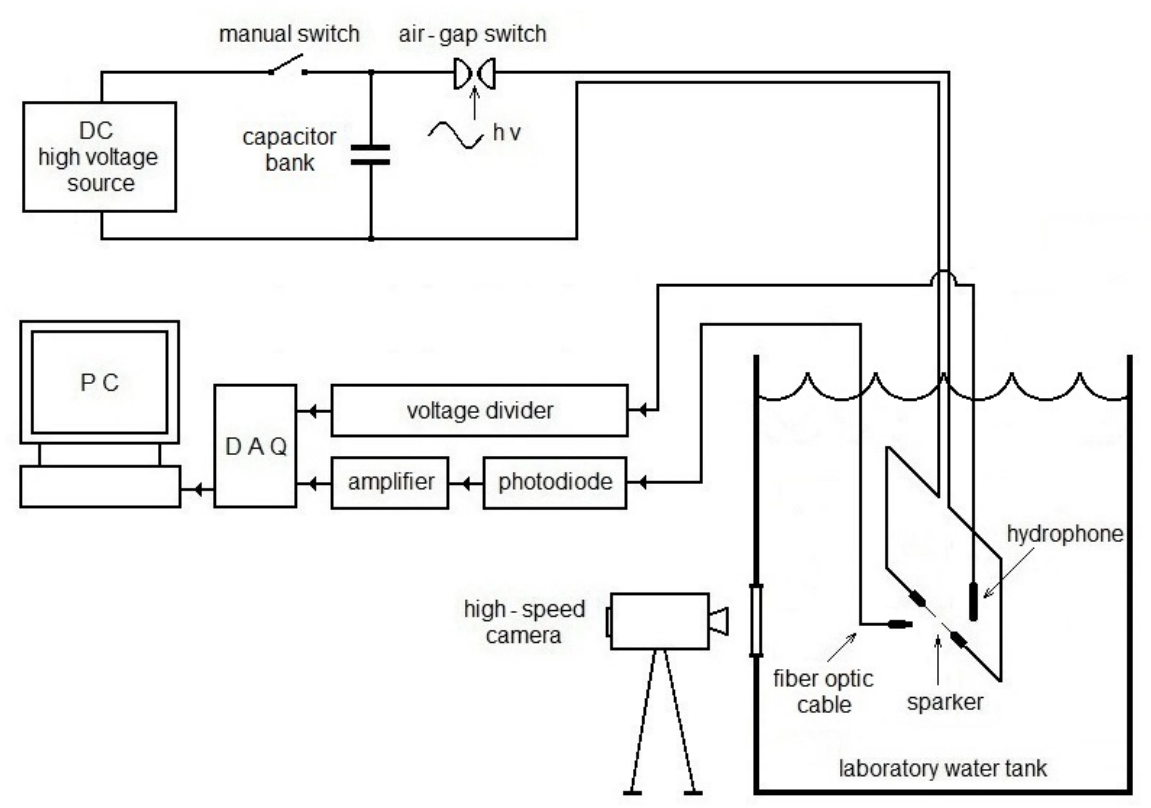

FIGURE 1. Experimental setup used to generate oscillating bubbles and to record the optical and acoustic radiation from them (DAQ - data acquisition board, hv - high voltage).

The acoustic radiation was monitored using a Reson broadband hydrophone type TC 4034. The hydrophone was positioned with the sensitive element at the same depth as the sparker. The distance between the hydrophone acoustic centre and the sparker gap was $r=0.2 \mathrm{~m}$. The output of the hydrophone was connected via a divider $10: 1$ to the second channel of the $\mathrm{A} / \mathrm{D}$ converter.

Prior to the measurements reported here, a limited number of high-speed camera records were taken with framing rates ranging $2800-3000 \mathrm{fps}$ (frames per second). A more detailed description of the experimental setup is given in an earlier work 19 .

In the experiments, a larger number of almost spherical bubbles freely oscillating in a large expanse of liquid were successively generated. The size of these bubbles, as described by the first maximum radius $R_{M 1}$, ranged $18.5-56.5 \mathrm{~mm}$, and the bubble oscillation intensity, as described by the nondimensional peak pressure in the first acoustic pulse $p_{z p 1}=\left(p_{p 1} / p_{\infty}\right)\left(r / R_{M 1}\right)$, ranged from 24 to 153 . Here $p_{p 1}$ is the peak pressure in the first acoustic pulse $p_{1}(t), p_{\infty}$ is the ambient (hydrostatic) pressure at the place of the sparker, and $r$ is the hydrophone distance from the sparker centre. Both $R_{M 1}$ and $p_{z p 1}$ were determined in each experiment from the respective pressure record, using an iterative procedure described in [21].

\section{Results AND DisCUSSION}

Examples of several frames from a film record taken with a high-speed camera are given in Figure 2 The first frame was taken in the bubble growth phase. The second to sixth frames correspond to the first contraction phase, and the seventh and eighth frames correspond to the first expansion phase. In the frames taken in the growth and first contraction phases, the glowing plasma core in the bubble interior can be seen. The small bright objects floating in the vicinity of the bubble are plasma packets [19.

The variation of the bubble radius $R$ with time $t$ is shown in Figure 3. The experimental data were determined from the frames, as are the data shown in Figure 2. As the spark-generated bubble is not ideally spherical (it is slightly elongated in the vertical direction), the data points represent an average from two perpendicular directions - one in the horizontal direction and the other in the vertical direction. The individual frames in Figure 2 can be traced to the corresponding points on the plot of bubble radius vs. time, given in Figure 3 .

The record of the optical radiation (represented by voltage $u(t)$ at the output of the optical detector) consists of a pulse $u_{0}(t)$ that is radiated during the electric discharge and the subsequent explosive bubble growth, and the pulse $u_{1}(t)$ that is radiated during the first bubble contraction and the subsequent bubble expansion [19]. The dynamic range of the optical detector (the photodiode, amplifier, A/D converter) was not sufficiently high to record both $u_{0}(t)$ and $u_{1}(t)$ in a single experiment with good fidelity. Two sets of experiments were therefore performed. The first set of experiments was aimed at recording the pulse $u_{0}(t)$ undisturbed, and the second set of experiments was 


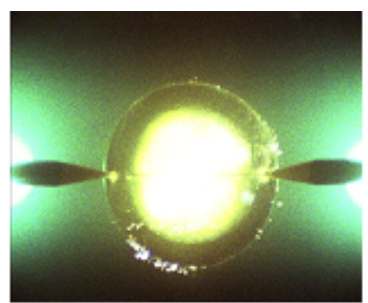

$t=2.38 \mathrm{~ms}$

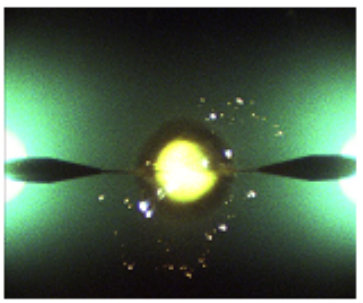

$t=8.09 \mathrm{~ms}$

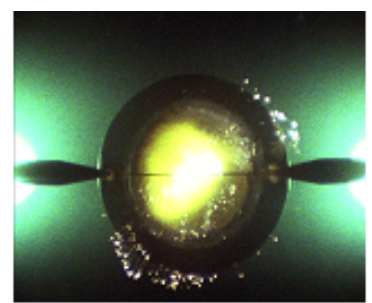

$t=4.52 \mathrm{~ms}$

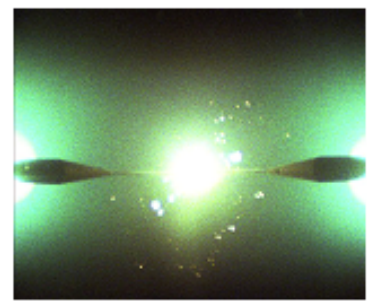

$t=8.45 \mathrm{~ms}$

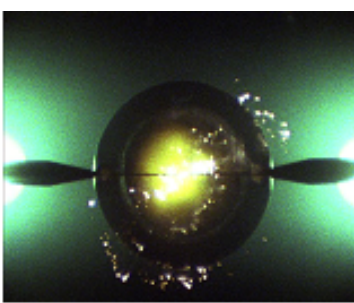

$t=5.95 \mathrm{~ms}$

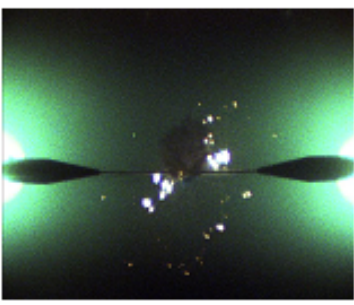

$t=8.81 \mathrm{~ms}$

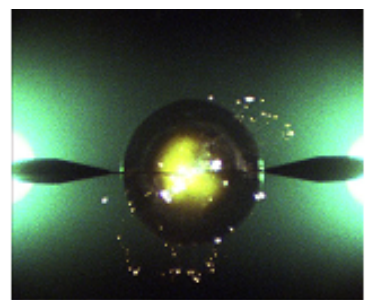

$t=7.38 \mathrm{~ms}$

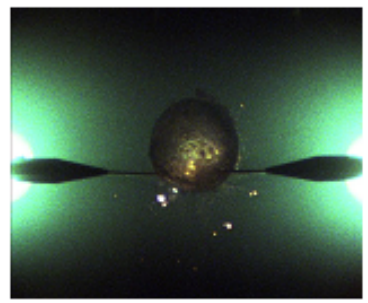

$t=10.59 \mathrm{~ms}$

FiguRE 2. Selected frames from a film record of a spark-generated bubble. The bubble is $R_{M 1}=51.5 \mathrm{~mm}$ in size, and oscillates with intensity $p_{z p 1}=70.3$. The times below each frame refer to the time origin, which is set at the instant of liquid breakdown. The spots of light on the sides of the frames are due to the illuminating lamps.

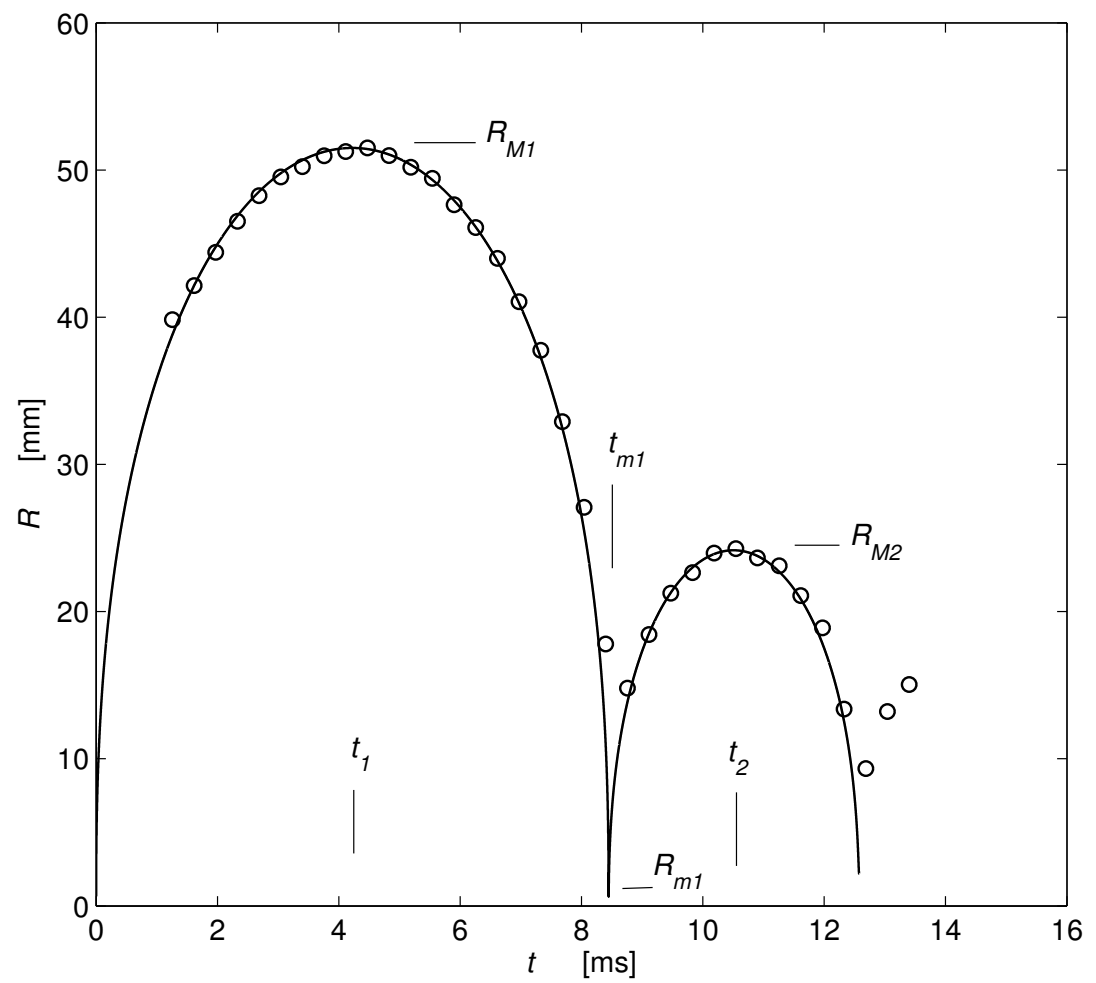

FiguRE 3. Variation of the bubble radius $R$ with time $t$ : ' $\circ$ ' experimental data, '-' fit to the experimental data (in the vicinity of $t_{m 1}$ this fit is only very approximate). The time origin is set at the instant of liquid breakdown. The time at which the bubble attains the first maximum radius $R_{M 1}$ is denoted as $t_{1}$; the time at which the bubble is compressed to the first minimum radius $R_{M 1}$ is denoted as $t_{m 1}$; and the time when the bubble attains the second maximum radius $R_{M 2}$ is denoted as $t_{2}$. The growth phase is defined to be within the interval $\left(0, t_{1}\right)$, the first contraction phase is within the interval $\left(t_{1}, t_{m 1}\right)$, and the first expansion phase is within the interval $\left(t_{m 1}, t_{2}\right)$. 


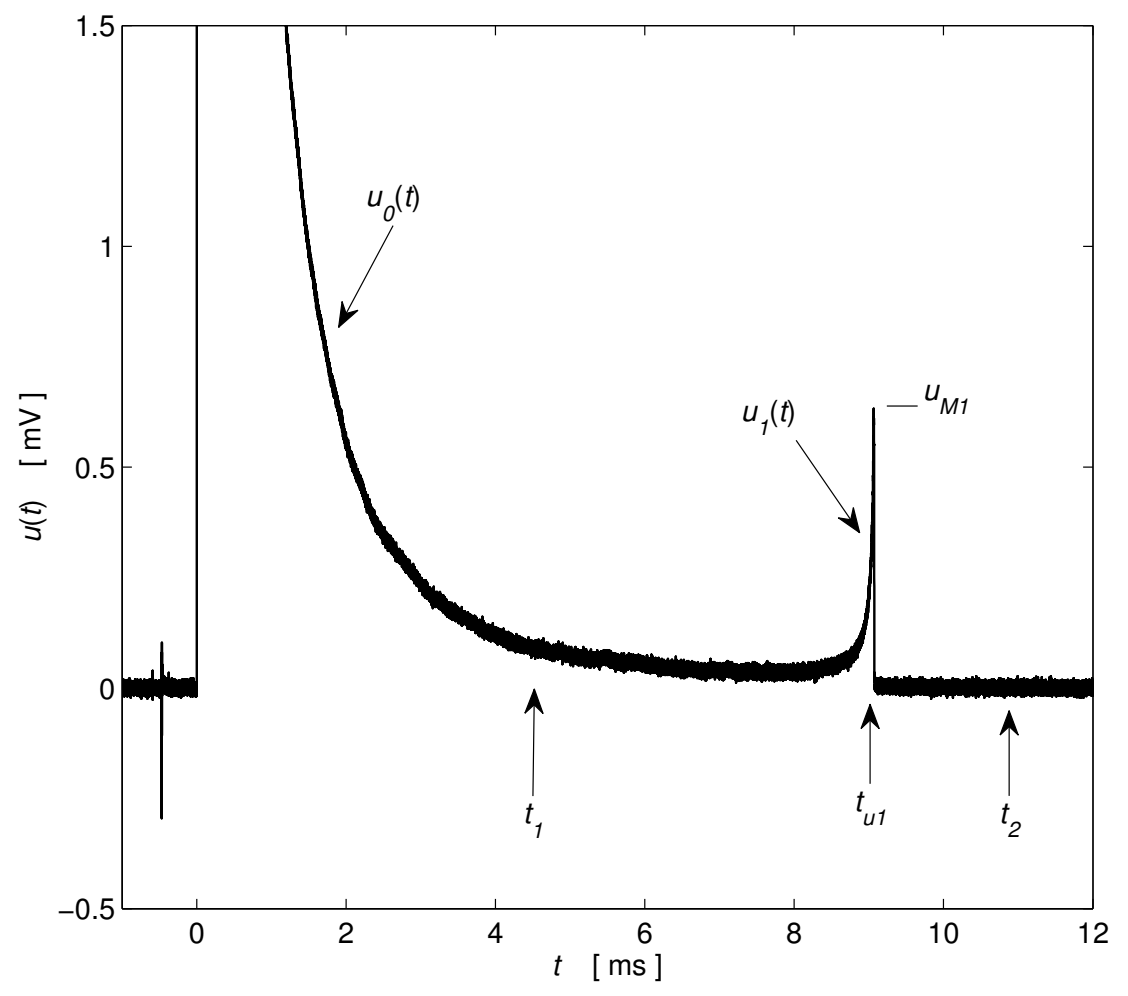

Figure 4 . Voltage $u(t)$ at the output of the optical detector. The spark-generated bubble is $R_{M 1}=55.2 \mathrm{~mm}$ in size, and oscillates with intensity $p_{z p 1}=153.2$. In this figure, the time origin is set at the instant of liquid breakdown, and this instant coincides with the beginning of the steep growth of the initial pulse $u_{0}(t)$. The time at which the bubble attains the first maximum radius $R_{M 1}$ is denoted as $t_{1}$, and the time when the bubble attains the second maximum radius $R_{M 2}$ is denoted as $t_{2}$. Pulse $u_{0}(t)$ is defined to be within the interval $\left(0, t_{1}\right)$, and pulse $u_{1}(t)$ is defined to be within the interval $\left(t_{1}, t_{2}\right)$. The blip before $t=0$ is a noise due to the air-gap switch.

aimed at recording the pulse $u_{1}(t)$ with acceptable noise. A link between the two sets of experiments is achieved by using statistical averages from the first set of records to compute the respective values for the second set of records.

An example of the optical pulse $u_{1}(t)$ from the second set of experiments is given in Figure 4. In Figure 4 the pulse $u_{0}(t)$ is clipped due to the limited dynamic range of the optical detector. The maximum value of pulse $u_{1}(t)$ is denoted as $u_{M 1}$, and the time of its occurrence is denoted as $t_{u 1}$. As can also be seen in Figure 4, the optical radiation from the bubble decreases rapidly to zero after $t_{u 1}$.

Another interesting fact that can be observed in Figure 4 is the occurrence of optical radiation from the bubble during the whole first oscillation, i.e., in the interval lasting approximately $\left(0, t_{u 1}\right)$. As can be observed in the photographs presented in Figure 2 , the source of this persisting optical radiation is the plasma core. It can also be seen in these photographs that the bubble interior is filled with two substances. First, there is some transparent matter, which is most probably hot water vapour. Second, there is opaque plasma at the bubble centre. The existence of this hot plasma core during the whole first bubble oscillation, i.e., even long after the electric discharge has terminated (in the case of the experimental data shown in Figure 4 the electric discharge lasted approximately only $0.5 \mathrm{~ms}$ ) is an astonishing phenomenon, observed already by Golubnichii et al. [9, 10. Golubnichii et al. called this outlasting plasma core "long-living luminescence formations". Similar long-lasting optical radiation has also been observed by Baghdassarian et al. [18. Baghdassarian et al. explain this radiation as the "luminescence from metastable atomic and molecular states injected into the water during or just after the plasma flash, which then recombine very slowly". However, it can be seen in Figure 2 that the light was emitted from the bubble interior, and not from the surrounding water. A direct comparison of Figure 4 with Figure 1 in [18] can also be used as further proof that in both spark-generated and laser-generated bubbles the glowing plasma core is present in their interior throughout the first bubble oscillation. And since there is no discharge current flowing through the laser-generated bubbles, it follows that the persisting plasma core in spark-generated bubbles is not due to a persisting discharge current.

Under the assumption that the hot plasma core in the bubble centre radiates as a black-body (this 


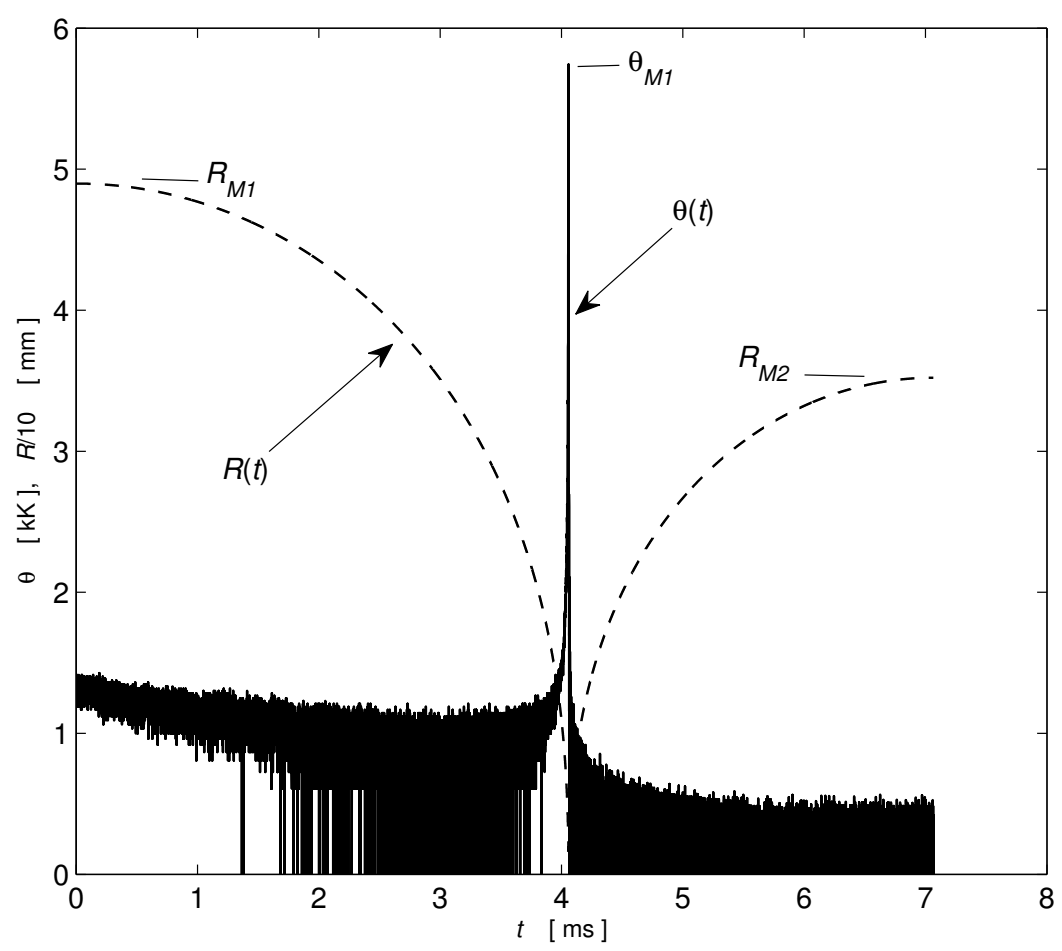

Figure 5. Time variation of the plasma core surface temperature $\Theta$ and of the bubble wall radius $R$. The size of the experimental bubble is $R_{M 1}=49.0 \mathrm{~mm}$, and the bubble oscillation intensity is $p_{z p 1}=142.1$. The time origin is set at the instant when the bubble radius equals $R_{M 1}$, i.e., it coincides with $t_{1}$. The maximum surface temperature of the plasma core at the first bubble contraction is denoted as $\Theta_{M 1}$.

assumption is justified, e.g., by the results published in [12]), an equation enabling the determination of the plasma surface temperature $\Theta(t)$ has been derived in [19]. The derivation is based on the StefanBoltzman Law, the equation of energy partition during the electric discharge, the time variation of the bubble radius $R(t)$, and the voltage $u(t)$ at the output of the optical detector. Particularly, for the voltage record $u_{1}(t)$ from the second set of experiments the corresponding temperature $\Theta(t)$ is given by the following equation

$$
\Theta^{4}(t)=\frac{\left\langle\Theta_{M 0}\right\rangle^{4}\left\langle R_{M 0}\right\rangle^{2}}{\left\langle u_{M 0}\right\rangle} \frac{u_{1}(t)}{R_{p}^{2}(t)} .
$$

Here $u_{M 0}$ is the maximum voltage in pulse $u_{0}(t)$, and this voltage corresponds to the bubble radius $R_{M 0}$. The surface temperature of the plasma, when the bubble during its growth attains radius $R_{M 0}$, is $\Theta_{M 0}$. The angle brackets \langle\rangle denote the average values on the first set of experiments. For a given bubble size $R_{M 1}$, these average values can be computed using the regression lines and the polynomial derived in [19]: $\left\langle\Theta_{M 0}\right\rangle=-0.11 R_{M 1}+17.4[\mathrm{kK}, \mathrm{mm}]$, $\left\langle R_{M 0}\right\rangle=0.1836 R_{M 1}$, and $\left\langle u_{M 0}\right\rangle=1.25 \cdot 10^{-4} R_{M 1}^{2}$ $[\mathrm{V}, \mathrm{mm}]$.

In (1), $R_{p}$ is the radius of the light-emitting hot plasma core. An estimate of radius $R_{p}$ can be obtained from knowledge of the bubble wall radius $R$ and of the volume that the plasma core occupies in the bubble interior. Denoting the reduction factor as $q(q<1)$, then $R_{p}=q R$. The variation of the bubble wall radius $R$ with time can be computed using a theoretical bubble model. The exact value of reduction factor $q$ is not known at present. A value of $q=0.2$ has been used in [19] for the region in the vicinity of the maximum bubble radius $R_{M 1}$. This value of $q$ has been estimated from high speed film records of the oscillating bubbles. When photographs of the bubble (e.g., in Figure 22) are inspected, it can be seen that during the contraction phase both the transparent vapour and the opaque plasma are gradually compressed, and remain mutually separated. The proportion of vapour and plasma changes in such a way that the value of reduction factor $q$ is increasing in the final stages of the contraction phase (the volume occupied by plasma decreases more slowly at later times of the contraction phase than the volume of vapour). Unfortunately, no estimate of $q$ can be made from the high speed camera frames for the important time interval covering the closest surroundings of instant $t_{u 1}$, when the bubble is compressed to its first minimum radius $R_{m 1}$. The reason why no estimate of $q$ can be obtained in this interval is that the frames were overexposed by the intensive light emitted from the bubble there (see the sixth frame in Figure 2). However, Ohl [13] has studied the luminescence from 


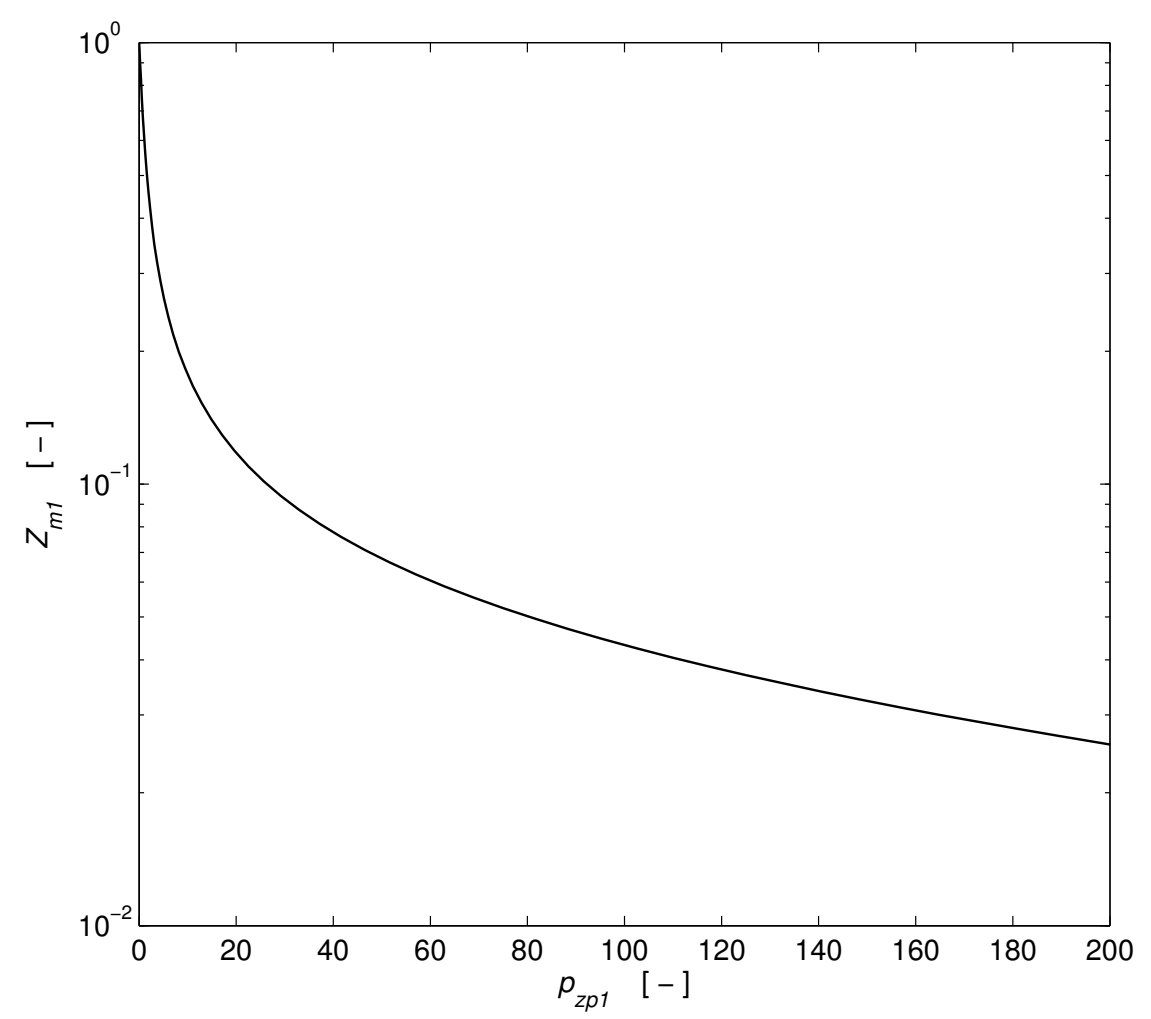

FiguRE 6 . The scaling function $Z_{m 1}=f\left(p_{z p 1}\right)$ computed with Herring's modified model of an ideal gas bubble oscillating in a large expanse of liquid.

a laser-generated bubble and gives (Figure 6 in 13) the size of the plasma core. The bubble studied by Ohl was $R_{M 1}=0.8 \mathrm{~mm}$ in size, and the radiating plasma core at the maximum contraction of the bubble had dimensions of $31 \mu \mathrm{m}$ (horizontal diameter) and $44 \mathrm{\mu m}$ (vertical diameter). This gives the mean value of the first minimum plasma core radius $R_{p m 1}=14 \mu \mathrm{m}$. When expressed in non-dimensional form, one obtains $Z_{p m 1}=R_{p m 1} / R_{M 1}=0.023$. An estimate of the nondimensional first minimum bubble wall radius given in [19] is $Z_{m 1}=R_{m 1} / R_{M 1}=0.03$. This gives an estimate of the reduction factor $q=Z_{p m 1} / Z_{m 1} \approx 0.8$, and this is the value of $q$ that will be used in this work for the closest surroundings of $R_{m 1}$.

An example of the variation of the plasma core surface temperature $\Theta$ with time $t$ during the first bubble contraction and the subsequent expansion, as computed with (1), is given in Figure 5. Equation (1) has been derived under the assumption that the plasma core is a black-body radiator. This assumption seems to be correct in those instants when the pressure and temperature in the bubble interior are high. And this is fulfilled only in the vicinity of $t_{u 1}$. Hence the computed temperature $\Theta(t)$ shown in Figure 5 is correct only in the vicinity of the maximum value $\Theta_{M 1}$. In other instants, the computed temperatures represent just a rough estimate.

In (1), only voltages $u_{1}(t)$ and $u_{M 0}$ are measured directly. Radii $R(t)$ and $R_{M 0}$ are computed using a theoretical model. In this work, Herring's modified model of a bubble freely oscillating in a compressible liquid far from boundaries is used [21]. In this model, the bubble is assumed to be an ideal gas bubble which behaves adiabatically. However, it has been shown earlier, by evaluating experimental pressure records [22], that spark-generated and laser-generated bubbles are vapour bubbles. This means that liquid evaporation and vapour condensation at the bubble wall play an important role during the oscillation of the bubble. It has been further shown by evaluating experimental optical records [19, that spark-generated and laser-generated bubbles do not behave adiabatically. Finally, in real spark-generated and laser-generated bubbles there are energy losses, the nature of which has not yet been clarified 19. They are therefore not encompassed in the theoretical model used to compute $R(t)$.

However, it can be shown [23], that during an interval lasting for almost all the time of bubble oscillation (except for short intervals at the beginning of the bubble growth and in the final stages of bubble contraction), the bubble wall motion is governed by the transform of the bubble potential energy into liquid kinetic energy, and vice versa. And neither the bubble potential nor the liquid kinetic energy depends on any of the processes mentioned above. That is, these energies and their transforms do not depend on the compressibility of the liquid, the liquid evap- 
oration and the vapour condensation at the bubble wall, an adiabatic assumption concerning the bubble thermal behaviour, or on any energy losses from the bubble. The computed variation of $R(t)$ (and thus also the computed values of $R_{M 0}$ ) may therefore be considered to be relatively correct in this long interval. There is only a very short time interval in the vicinity of the minimum radius $R_{m 1}$, in which all the above-mentioned processes manifest themselves and play an important role. Unfortunately, both the experimental description and the theoretical description of the bubble are still insufficient precisely in this short interval.

It is a very difficult task to make an experimental determination of $R_{m 1}$. For example, the framing rate of the high speed camera used here was too low to enable $R_{m 1}$ to be determined. Even those researchers who have succeeded making an experimental observation of $R_{m 1}$ encountered extreme difficulties. It is therefore not surprising that few experimentally determined values of $R_{m 1}$ are given in the literature on the oscillation of spark-generated and laser-generated bubbles and those values that have been given vary greatly. In addition, unfortunately, the authors usually do not provide further data enabling the computation of $p_{z p 1}$. Because knowledge of the variation of $R_{m 1}$ with $p_{z p 1}$ is important in this work, a theoretical computation of $R_{m 1}$ is preferred in the following.

The following procedure will be used to determine the value of $R_{p m 1}$, which is needed in (1) for the computation of temperature $\Theta_{M 1}$. First, using Herring's modified bubble model [21], the scaling function $Z_{m 1}=R_{m 1} / R_{M 1}=f\left(p_{z p 1}\right)$ is determined. The computed scaling function $Z_{m 1}=f\left(p_{z p 1}\right)$ is shown in Figure 6. The physical constants used in the computation are: water density $\rho=10^{3} \mathrm{~kg} \mathrm{~m}^{-3}$, the polytrophic exponent of the gas in a bubble interior $\gamma=1.25$, the velocity of sound in water $c=1480 \mathrm{~m} \mathrm{~s}^{-1}$, and the hydrostatic pressure $p_{\infty}=125 \mathrm{kPa}$.

The scaling function $Z_{m 1}=f\left(p_{z p 1}\right)$ has been computed for $p_{z p 1}$ ranging from 1 to 200 , as this is the range of the bubble oscillation intensities encountered in experiments with spark-generated bubbles [21]. In the experiments that are analysed in this work, parameters $R_{M 1}$ and $p_{z p 1}$ have been determined for each record $u_{1}(t)$. Then, using the scaling function $Z_{m 1}\left(p_{z p 1}\right)$ given in Figure 6, an estimate of the first minimum radius $R_{m 1}=Z_{m 1} R_{M 1}$ can be obtained for each experimental record $u_{1}(t)$. An estimate of the corresponding plasma core radius is then $R_{p m 1}=0.8 R_{m 1}$. Thus, using the measured values of $u_{M 1}, R_{M 1}$, and $p_{z p 1}$ from the second set of experiments and the average values of $\left\langle\Theta_{M 0}\right\rangle,\left\langle R_{M 0}\right\rangle$, and $\left\langle u_{M 0}\right\rangle$ determined for a given bubble size $R_{M 1}$ from the regression lines and the polynomial given above, temperature $\Theta_{M 1}$ can be computed. The values of $\Theta_{M 1}$ determined in this way for different bubble sizes $R_{M 1}$ and for different bubble oscillation intensities $p_{z p 1}$ are displayed in Figures 7 and 8 .
Figures 7 and 8 show that the temperatures $\Theta_{M 1}$ determined in this work range c. $4300-8700 \mathrm{~K}$. The regression lines for the mean values of temperature $\Theta_{M 1}$ in dependence on $R_{M 1}$ and $p_{z p 1}$ are $\left\langle\Theta_{M 1}\right\rangle=$ $-0.04 R_{M 1}+7.57[\mathrm{kK}, \mathrm{mm}]$ and $\left\langle\Theta_{M 1}\right\rangle=0.01 p_{z p 1}+$ $4.61[\mathrm{kK},-]$. It can be seen that the temperatures $\Theta_{M 1}$ vary only moderately with bubble sizes and with the oscillation intensities. Whereas the small variation of $\Theta_{M 1}$ with $R_{M 1}$ is as expected (the bubbles studied here have sizes for which it can be assumed that the scaling law is valid [24]), the small dependence of $\Theta_{M 1}$ on $p_{z p 1}$ is surprising. It shows that the plasma in a bubble behaves rather autonomously, i.e., the plasma temperature varies very little with the pressure in the interior of the bubble. This can be shown easily in the following numerical examples. Let us denote the peak pressure at a bubble wall at the final stages of the first contraction as $P_{p 1}$. A rough estimate of the value of $P_{p 1}$ can be obtained from the experimentally determined values of $p_{z p 1}$ and the computed scaling function $Z_{m 1}=f\left(p_{z p 1}\right)$ displayed in Figure 6. At a bubble wall it holds that $r=R_{m 1}$ and $p_{p 1}=P_{p 1}$. After substituting these equalities into the definition formula of $p_{z p 1}$ given in Section ?? (i.e., into the relation $\left.p_{z p 1}=\left(p_{p 1} / p_{\infty}\right)\left(r / R_{M 1}\right)\right)$, one obtains $p_{z p 1}=P_{p 1} Z_{m 1} / p_{\infty}$. This equation can be rearranged to give $P_{p 1}=p_{z p 1} p_{\infty} / Z_{m 1}$. Then, for $p_{z p 1}=25$ and $p_{\infty}=125 \mathrm{kPa}$ it can be read from Figure 6 that $Z_{m 1} \approx 0.1$ and thus $P_{p 1} \approx 31 \mathrm{MPa}$. For $p_{z p 1}=150$ and $p_{\infty}=125 \mathrm{kPa}$, it can be read from Figure 6 that $Z_{m 1} \approx 0.032$ and thus $P_{p 1} \approx 586 \mathrm{MPa}$ It follows then that the variation of the peak pressure at the bubble wall $P_{p 1}$ from $31 \mathrm{MPa}$ to $586 \mathrm{MPa}$, i.e., by a factor of approximately 19, is accompanied by variation of the mean temperature $\left\langle\Theta_{M 1}\right\rangle$ from $4860 \mathrm{~K}$ to $6110 \mathrm{~K}$, i.e. only by a factor of 1.26 (the temperatures $\left\langle\Theta_{M 1}\right\rangle$ have been computed using the regression lines given above). The differences in the variation of $P_{p 1}$ and $\Theta_{M 1}$ provide surprising evidence about the autonomous behaviour of the plasma in the bubble interior.

Finally in this Section, the temperatures $\Theta_{M 1}$ given in Figure 7 can be compared with the experimental results of other researchers obtained with bubbles oscillating in water under ordinary laboratory conditions and far from boundaries. The first rough estimate of $\Theta_{M 1}$ can be derived from data published by Buzukov and Teslenko [8]. These researchers studied bubbles generated by a laser, and found that the optical radiation associated with the first optical pulses $u_{1}(t)$ had continuum spectra with spectral maxima occurring in the range $375-440 \mathrm{~nm}$. Using Wien's Law, it can be calculated that the temperatures $\Theta_{M 1}$ ranged 6600-7700 K. Unfortunately, the sizes of the bubbles generated in these experiments are not available. Golubnichii et al. [5] studied bubbles generated by an exploding wire technique, and found that the maximum in the spectrum of the optical radiation associated with the first optical pulse $u_{1}(t)$ lies approximately 


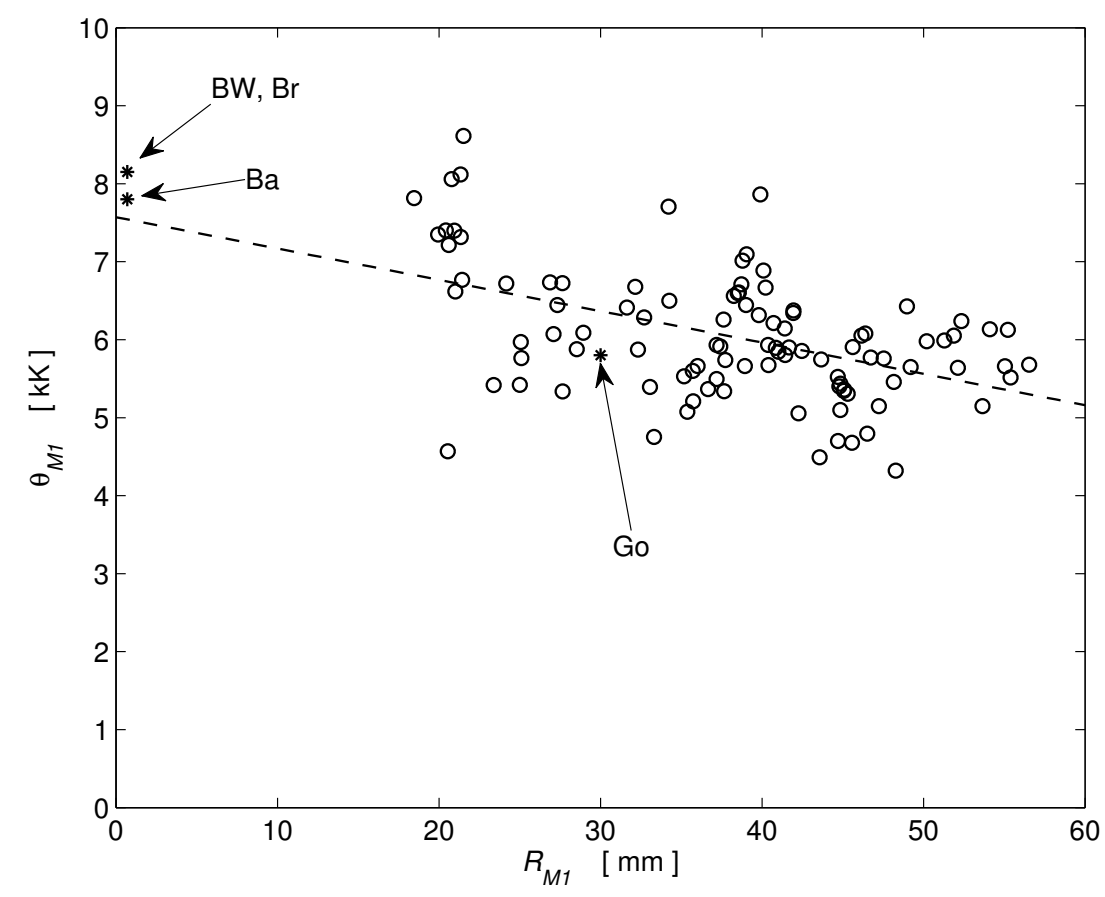

FigURE 7. Variation of experimentally determined maximum surface temperatures of the plasma core during the first bubble contraction $\Theta_{M 1}$ with bubble size $R_{M 1}$ : ' $\circ$ ' — the values of $\Theta_{M 1}$ determined in this work, ' $*$ ' — the values of $\Theta_{M 1}$ determined in 5] (Go), 12] (Ba), 14] (BW), 15] (Br); these values are discussed at the end of this section.

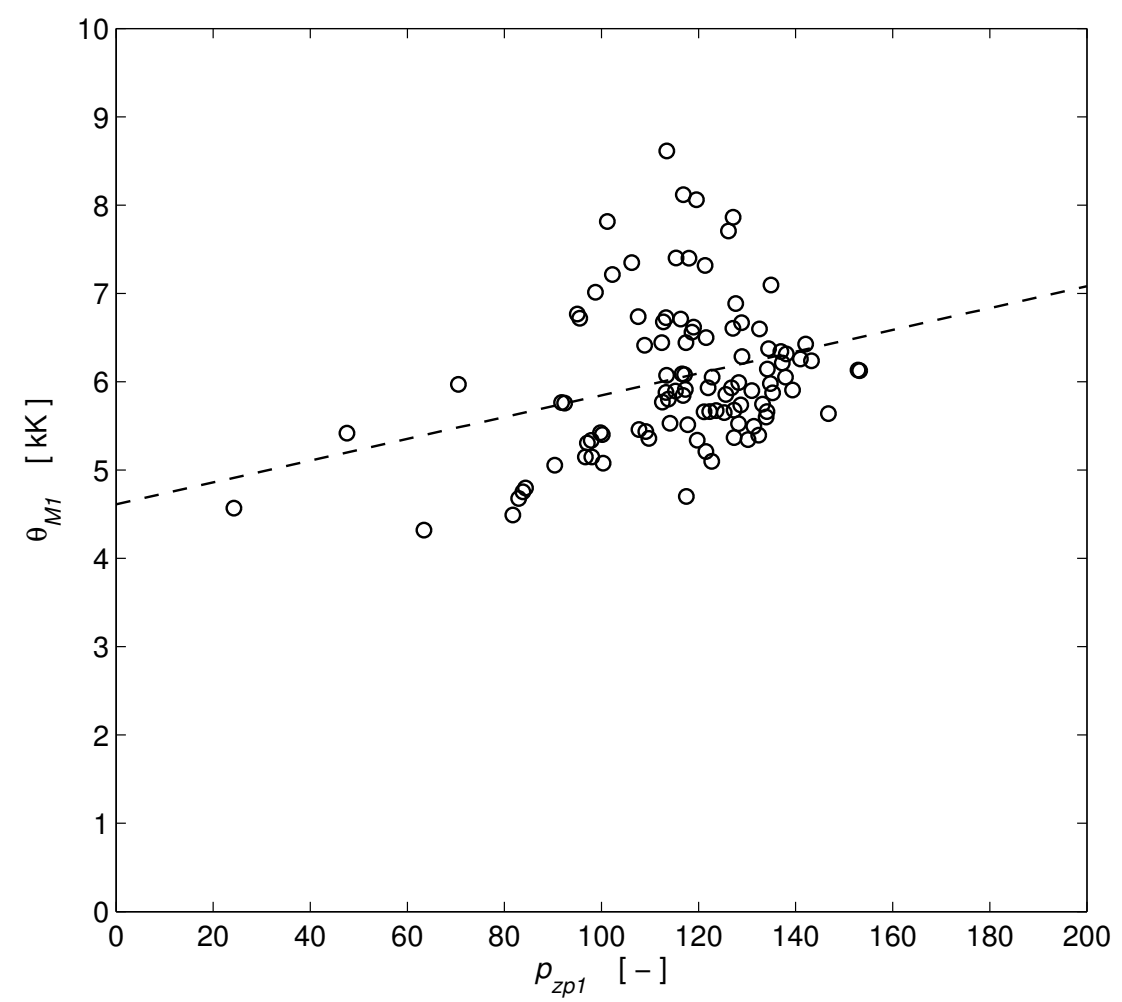

FIGURE 8. Variation of the experimentally determined maximum surface temperatures of the plasma core during the first bubble contraction $\Theta_{M 1}$ with bubble oscillation intensity $p_{z p 1}$. 
at $500 \mathrm{~nm}$. Then, using Wien's Law, temperature $\Theta_{M 1}=5800 \mathrm{~K}$ is obtained. In this case, the size of the bubbles was $R_{M 1}=30 \mathrm{~mm}$. Baghdassarian et al. 12 studied laser-generated bubbles $R_{M 1}=0.6-0.8 \mathrm{~mm}$ in size, and from the gated optical spectra they determined that $\Theta_{M 1}=7800 \mathrm{~K}$. Finally, Brujan and Williams [14, and Brujan et al. 15] also studied lasergenerated bubbles, and from the gated optical spectra they determined that $\Theta_{M 1}=8150 \mathrm{~K}$. In this case, the bubble sizes $R_{M 1}$ ranged from $0.65 \mathrm{~mm}$ to 0.75 $\mathrm{mm}$. Unfortunately, no data are given in the quoted references to enable the bubble oscillation intensity to be determined.

It should be pointed out that the temperatures obtained in this work represent instantaneous values, and can be determined for any instant at which the assumption of black-body radiation is valid. In the works of other authors [5, 8, 12, 14, 15], however, no exact instant is given when the temperature was determined. It is only assumed that the measured temperature corresponds to the maximum bubble contraction. However, when the autonomous plasma behaviour is taken into account, this assumption may not always be correct, and experimental verification is still required. The temperatures measured in this work can also be assigned to particular experiments. This is again in contrast with other works [12, 14, 15, where the spectra have been averaged over 25-50 experiments. Although care was taken in those works to average only the spectra corresponding to bubbles of almost the same size, the variation of the temperature with the bubble oscillation intensity (and maybe even with some other as yet unknown factors) has been lost during the averaging procedure.

\section{Conclusions}

The surface temperatures of the plasma core inside spark-generated bubbles in the final stages of the first contraction phase have been determined experimentally. It was found that these temperatures range $4300 \mathrm{~K}$ to $8700 \mathrm{~K}$. As the statistical averages from another set of experiments were used in the computations, the method employed gives only approximate results. Nevertheless, it has been shown that the values that were obtained are in good agreement with the temperatures published by other researchers. However, unlike the results presented by other researchers, the results presented here were obtained on a large set of bubbles of different sizes that oscillated with different intensities.

It has also been shown that the plasma inside bubbles behaves rather autonomously, i.e., the surface temperature of the plasma varies only very little with the pressure at the bubble wall. The average maximum surface temperature $\left\langle\Theta_{M 1}\right\rangle$ increases moderately with bubble oscillation intensity $p_{z p 1}$, and decreases moderately with bubble size $R_{M 1}$. In any case, it can be concluded that plasma in bubbles behaves rather differently from the ideal gas that has so often been considered.

The experimental method described in this work offers an alternative to spectral methods. As has been discussed here, the method has certain advantages and disadvantages. It can be anticipated that, after further improvements, the precision of this method may be increased.

\section{ACKNOWLEDGEMENTS}

This work has been supported by the Ministry of Education of the Czech Republic as research project MSM 245100304. The experimental part of this work was carried out during the author's stay at the Underwater Laboratory of the Italian Acoustics Institute, CNR, Rome, Italy. The author wishes to thank Dr. Silvano Buogo from this laboratory for his very valuable help in preparing the experiments.

\section{REFERENCES}

[1] S.J. Shaw, W.P. Schiffers, D.C. Emmony.

Experimental observation of the stress experienced by a solid surface when a laser-created bubble oscillates in its vicinity. J. Acoust. Soc. Am. 110, 1822-1827, 2001. DOI:10.1121/1.1397358

[2] A. Jayaprakash, C.-T. Hsiao, G. Chahine. Numerical and experimental study of the interaction of a sparkgenerated bubble and a vertical wall. Trans. ASME J. Fluid Eng. 134, 031301, 2012. DOI:10.1115/1.4005688

[3] V. Sboros. Response of contrast agents to ultrasound. Adv. Drug Deliver. Rev. 60, 1117-1136, 2008. DOI:10.1016/j.addr.2008.03.011

[4] T.G. Leighton, C.K. Turangan, A.R. Jamaluddin, G.J Ball, P.R. White. Prediction of far-field acoustic emissions from cavitation clouds during shock wave lithotripsy for development of a clinical device. Proc. $R$. Soc. A 469, 20120538, 2013. DOI:10.1098/rspa.2012.0538

[5] P.I. Golubnichii, V.M. Gromenko, A.D. Filonenko. The nature of an electrohydrodynamic sonoluminescence impulse (in Russian). Zh. Tekh. Fiz. 50, 2377-2380, 1980.

[6] S.W. Fong, D. Adhikari, E. Klaseboer, B.C. Khoo. Interactions of multiple spark-generated bubbles with phase differences. Exp. Fluids 46, 705-724, 2009. DOI:10.1007/s00348-008-0603-4

[7] Y. Huang, H. Yan, B. Wang, X. Zhang, Z. Liu, K. Yan. The electro-acoustic transition process of pulsed corona discharge in conductive water. J. Phys. D: Appl. Phys. 47, 255204, 2014. DOI:10.1088/0022-3727/47/25/255204

[8] A.A. Buzukov, V.S. Teslenko. Sonoluminescence following the focusing of laser radiation into a liquid (in Russian). Pisma Zh. Tekh. Fiz. 14, 286-289, 1971.

[9] P.I. Golubnichii, V.M. Gromenko, Ju.M Krutov. Formation of long-lived luminescent objects under decomposition of a dense low-temperature water plasma (in Russian). Zh. Tekh. Fiz. 60, 183-186, 1990.

[10] P.I. Golubnichii, V.M. Gromenko, Ju.M. Krutov. Long-lived luminescent formations inside a pulsating cavern initiated by powerful energy emission in water (in Russian). Dokl. Akad. Nauk SSSR 311, 356-360, 1990. 
[11] L. Zhang, X. Zhu, H. Yan, Y. Huang, Z. Liu, K. Yan. Luminescence flash and temperature determination of the bubble generated by underwater pulsed discharge. Appl. Phys. Lett. 110, 034101, 2017. DOI:10.1063/1.4974452

[12] O. Baghdassarian, H.-C. Chu, B. Tabbert, G.A. Williams. Spectrum of luminescence from laser-created bubbles in water. Phys. Rev. Lett. 86, 4934-4937, 2001. DOI:10.1103/PhyRevLett.86.4934

[13] C.-D. Ohl. Probing luminescence from nonspherical bubble collapse. Phys. Fluids 14, 2700-2708, 2002. DOI:10.1063/1.1489682

[14] E.A. Brujan, G.A. Williams. Luminescence spectra of laser-induced cavitation bubbles near rigid boundaries. Phys. Rev. E 72, 016304, 2005. DOI:10.1103/PhysRevE.72.016304

[15] E.A. Brujan, D.S. Hecht, F. Lee, G.A. Williams. Properties of luminescence from laser-created bubbles in pressurized water. Phys. Rev. E 72, 066310, 2005. DOI:10.1103/PhysRevE.72.066310

[16] H.J. Park, G.J. Diebold. Generation of cavitation luminescence by laser-induced exothermic chemical reaction. J. Appl. Phys. 114, 064913, 2013. DOI:10.1063/1.4818516

[17] T. Sato, M. Tinguely, M. Oizumi, M. Farhat. Evidence for hydrogen generation in laser- or spark-induced cavitation bubbles. Appl. Phys. Lett. 102, 074105, 2013. DOI:10.1063/1.4793193
[18] O. Baghdassarian, B. Tabbert, G.A. Williams. Luminescence characteristics of laser-induced bubbles in water. Phys. Rev. Lett. 83, 2437-2440, 1999. DOI:10.1103/PhysRevLett.83.2437

[19] K. Vokurka, J. Plocek. Experimental study of the thermal behavior of spark-generated bubbles in water. Exp. Therm. Fluid Sci. 51, 84-93, 2013. DOI:10.1016/j.expthermflusci.2013.07.004

[20] K. Vokurka. Determination of temperatures in oscillating bubbles: experimental results. In: 22nd International Conference ENGINEERING MECHANICS 2016, Svratka, Czech Republic 9-12 May 2016 (conference proceedings: Institute of Thermomechanics, Academy of Sciences of the Czech Republic, v.v.i., Prague 2016, ISBN: 978-80-87012-59-8, ISSN: 1805-8248, editors: Igor Zolotarev, Vojtěch Radolf, pp. 581-584).

[21] S. Buogo, K. Vokurka. Intensity of oscillation of spark generated bubbles. J. Sound Vib. 329, 4266-4278, 2010. DOI:10.1016/j.jsv.2010.04.030

[22] K. Vokurka. A model of spark and laser generated bubbles. Czech. J. Phys. B38, 27-34, 1988. DOI:10.1007/BF01596516

[23] K. Vokurka. Significant intervals of energy transforms in bubbles freely oscillating in liquids. J. Hydrodyn. 29, 217-225, 2017. DOI:10.1016/S1001-6058(16)60731-X

[24] K. Vokurka. The scaling law for free oscillations of gas bubbles. Acustica 60, 269-276, 1986. 